\title{
Destroying Coherence in High-Temperature Superconductors with Current Flow
}

\author{
A. Kaminski, ${ }^{1}$ S. Rosenkranz, ${ }^{2}$ M. R. Norman, ${ }^{2}$ M. Randeria, ${ }^{3}$ Z. Z. Li, ${ }^{4}$ H. Raffy, ${ }^{5}$ and J. C. Campuzano ${ }^{6}$ \\ ${ }^{1}$ Ames Laboratory and Department of Physics and Astronomy, Iowa State University, \\ Ames, Iowa 50011, USA \\ ${ }^{2}$ Materials Science Division, Argonne National Laboratory, Argonne, Illinois 60439, USA \\ ${ }^{3}$ Department of Physics, The Ohio State University, Columbus, Ohio 43210, USA \\ ${ }^{4}$ Laboratoire de Physique des Solides, CNRS, Université Paris-Sud, 91405 Orsay Cedex, France \\ ${ }^{5}$ Laboratoire de Physique des Solides, CNRS, Université Paris-Sud, 91405 Orsay Cedex, France \\ ${ }^{6}$ Department of Physics, University of Illinois at Chicago, Chicago, Illinois 60607, USA \\ (Received 25 September 2015; revised manuscript received 10 February 2016; published 13 September 2016)
}

\begin{abstract}
The loss of single-particle coherence going from the superconducting state to the normal state in underdoped cuprates is a dramatic effect that has yet to be understood. Here, we address this issue by performing angle resolved photoemission spectroscopy measurements in the presence of a transport current. We find that the loss of coherence is associated with the development of an onset in the resistance, in that well before the midpoint of the transition is reached, the sharp peaks in the angle resolved photoemission spectra are completely suppressed. Since the resistance onset is a signature of phase fluctuations, this implies that the loss of single-particle coherence is connected with the loss of long-range phase coherence.
\end{abstract}

DOI: 10.1103/PhysRevX.6.031040

Subject Areas: Condensed Matter Physics, Strongly Correlated Materials, Superconductivity

\section{INTRODUCTION}

In the classic theory of superconductivity of Bardeen, Cooper, and Schrieffer [1], an underlying assumption is the presence of sharp quasiparticles in the normal state. In underdoped cuprates, this condition is violated in that the pseudogap phase is associated with broad, incoherent electronic excitations [2-4]. If spectral broadening arises from electron-electron scattering, then it might be tempting to argue that the closing of the superconducting gap leads to loss of single-particle coherence, since scattering processes that were gapped out below $T_{c}$ could become important above $T_{c}$. But precisely the opposite is seen in experiments. In overdoped cuprates where the superconducting gap indeed closes at $T_{c}$, coherent quasiparticles are seen to persist for temperatures well above $T_{c}$ [5]. But in the pseudogap phase, coherence is absent above $T_{c}$ despite the presence of a large energy gap that persists to a much higher temperature, $T^{*}[6]$.

A number of years ago, it was noted that the intensity of the quasiparticle peak increases upon cooling below $T_{c}$ in underdoped cuprates $[7,8]$ and its spectral weight tracks the superfluid density $[9,10]$, but the exact relation between a two-particle correlation function (the superfluid density)

Published by the American Physical Society under the terms of the Creative Commons Attribution 3.0 License. Further distribution of this work must maintain attribution to the author(s) and the published article's title, journal citation, and DOI. and a single-particle one (the presence of quasiparticles) is far from obvious.

Here, we advocate a new approach to study this important problem by performing angle resolved photoemission spectroscopy (ARPES) measurements in the presence of a transport current [11] that induces a resistive state in the sample below $T_{c}$ [12-16]. The idea here is to use current flow to destroy the superconducting state, distinct from simply raising the temperature above $T_{c}$, and then use spectroscopy to probe the question of singleparticle coherence of the electronic excitations.

Our main result is that the loss of coherent electronic excitations in underdoped cuprates is associated with onset of resistance, with the sharp peaks in the ARPES spectra completely suppressed well before the midpoint of the resistive transition. We argue that the onset of resistance occurs due to motion of vortices in a state with local superconducting order, and thus the loss of sharp quasiparticles is tied to the loss of long-range phase coherence in the superconductor.

Before getting into details of our analysis and its implications, we note that the methodology we introduce here-ARPES in the presence of current flow-has the promise of opening up new opportunities for probing quantum materials. The investigation of nonequilibrium states of quantum matter is still in its infancy, with pumpprobe spectroscopy (optics and ARPES) being the most commonly used technique for solid-state systems. The new methodology we develop here can be used to probe the single-particle spectroscopy of nonequilibrium steady states in the presence of current flow. This could lead to 
new insights into many different problems, for instance, (i) superconducting materials, especially since many applications necessarily involve current flow, (ii) charge density wave materials, where current flow leads to a depinning of the charge density wave, and (iii) correlated materials with complex phase diagrams, where current flow could alter the relative stability of competing phases.

The rest of the paper is organized as follows. Given that we introduce a completely new methodology, a significant fraction of the paper is devoted to a careful discussion of experimental issues. In Sec. II, we describe the samples and the device geometry. In Sec. III A, we estimate various effects related to current flow in the sample, including the effect of the resulting fields on ARPES and the important question of Joule heating (with details relegated to Appendixes A-E). We note that the analysis of Joule heating is central to our work, since only then can we prove that we observe the nontrivial effects of current flow below the sample $T_{c}$, rather than the known effects of heating the sample above $T_{c}$. We should also emphasize that our ARPES measurements are taken when the resistance of the samples with current flow is only one-third of the "zero-current" resistance above $T_{c}$; thus, Joule heating is small enough not to raise the sample temperature above $T_{c}$. In Sec. III B, we present the ARPES data in the presence of current flow and contrast the behavior observed in underdoped and overdoped cuprates. Finally, we conclude with some remarks on the broader implication of our results for cuprates.

\section{EXPERIMENTAL DETAILS}

We utilize thin $(\sim 500 \AA)$ films of $\mathrm{Bi}_{2} \mathrm{Sr}_{2} \mathrm{CaCu}_{2} \mathrm{O}_{8+\delta}$ (Bi2212) prepared by rf sputtering on $\mathrm{SrTiO}_{3}$ (STO) substrates. These samples possess ARPES and transport characteristics very similar to those of single crystals, but their small cross sections allow us to obtain high current densities of $\sim 10^{6} \mathrm{~A} / \mathrm{cm}^{2}$ using modest values of the current $(\leq 200 \mathrm{~mA})$. The films also display small signals from the structural superlattice distortion $(<3 \%$ intensity of the main band), thus simplifying the interpretation of ARPES data near the Brillouin zone boundary. The thin film samples are patterned into the shape of two large rectangular pads $\left(3 \times 2 \mathrm{~mm}^{2}\right)$ connected by a narrow bridge of width $\sim 250 \mu \mathrm{m}$ and length $1 \mathrm{~mm}$, as shown in Figs. 1(a) and 1(b). Two electrical contacts are made by evaporating gold onto those two pads on the top of the sample and then attaching a single copper wire to each with silver paste. Current is injected through such made contacts. The residual resistance of the sample-gold junction is measured ex situ in a four-point contact setup to be less than $20 \mathrm{~m} \Omega$. The current path is returned parallel to the sample in order to reduce the magnetic field and provide a ground plane to induce a uniform current through the sample, as shown in Fig. 1(a). The electrical insulation between the sample and the current return electrode is provided by the
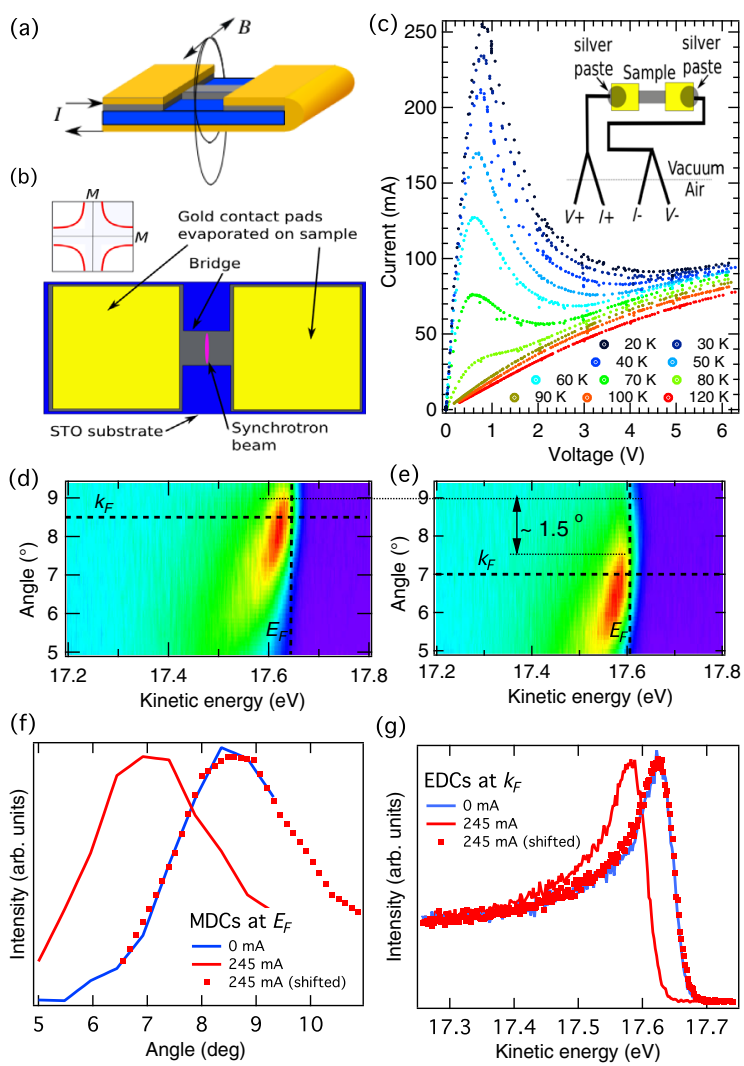

FIG. 1. (a) Schematic diagram of the measurement geometry. Gray area is the thin film Bi2212 sample, blue color marks the STO substrate, and gold color signifies the metallic contacts. (b) Schematic drawing (not to scale) of the sample, bridge, gold pads, and synchrotron beam. The inset is the 2D Brillouin zone, with $M \equiv(\pi, 0)$. (c) $I V$ characteristics at various temperatures for optimally doped (OP) Bi2212 sample. The inset shows the electrical connection to the sample inside the vacuum. (d),(e) ARPES intensity along the zone diagonal without (d) and with (e) a current flowing through a copper plate placed underneath the substrate. A small shift of $\sim 1.5^{\circ}$ is primarily due to the magnetic field generated by the current $(0.845 \mathrm{~V}, 245 \mathrm{~mA})$. Note that in this test configuration, the current is not flowing through the sample. The shift in the energy is due to the sample being in electrical contact with one of the current leads. $E_{F}$ and $k_{F}$ for each case are marked by dashed lines. (f) momentum distribution curves (MDCs) at $E_{F}$ with and without current, as in (d) and (e). A copy of the MDC with current (dotted curve) is shifted in momentum for a line shape comparison. (g) energy distribution curves (EDCs) at $k_{F}$ with and without current, as in (d) and (e). A copy of the EDC with current (dotted curve) is shifted in energy for a line shape comparison.

STO substrate. The substrate is nonconducting with a resistance larger then $40 \mathrm{M} \Omega$ (limit of our ohm meter) and, thus, does not contribute to the electrical transport. The two wires carrying the sample current are thermally anchored to the cold finger, then attached to two pairs (one pair for supplying the current to the sample and one pair for measuring the voltage drop) of thinner, long wires that are wrapped many times around both stages of the cold 
finger and connected to the electrical feedthrough. Such a configuration, illustrated in the inset of Fig. 1(c), minimizes the heat transport from the electrical feedthrough that is at room temperature through the wires to the sample, while minimizing the heat dissipation in the wires attached to the gold pads and maintaining the ability to measure the voltage relatively close to the sample. The power supply operating in constant voltage mode is connected to the current leads and a digital voltmeter is connected to the voltage leads. The $I V$ curves are measured with the UV beam switched off, and we do not observe any changes in the current nor voltage values when the UV beam is switched on for the ARPES measurements. A small aluminum pin of similar shape is glued to the top of the bridge and used to cleave its surface in situ. The thickness of the bridge and thus its resistance varies from sample to sample due to cleaving and is roughly of the order of $500 \AA$.

ARPES measurements are carried out using our SES50 movable electron energy analyzer and $4 \mathrm{~m}$ normal incidence monochromator on the U1 undulator beam line at the Synchrotron Radiation Center in Wisconsin. A movable analyzer allows the acquisition of data where the energy gap is maximal (antinodal regions of the zone), and a chemical potential reference where the gap is zero (zone diagonals), without moving the sample with respect to the photon beam. The sample is mounted in the geometry where the $\mathrm{Cu}-\mathrm{O}$ bond direction is parallel to the polarization plane of the photons, with a photon energy of $22 \mathrm{eV}$ employed.

\section{RESULTS AND DISCUSSION}

\section{A. ARPES spectroscopy in the presence of current}

When current is flowing in the sample, electric and magnetic fields exist in the vacuum, which deflect the outgoing photoelectrons. We test the effects of these fields empirically by passing a substantial current through a copper plate insulated from the sample and mounted just underneath. The effects of the current are illustrated in Figs. 1(d) and 1(e), where a $\sim 1.5^{\circ}$ deflection is observed with an applied current. The MDCs at $E_{F}$ and EDCs at $k_{F}$ for data with and without the current flow are shown in Figs. 1(f) and 1(g). $E_{F}$ is determined by integrating EDCs along the momentum cuts and fitting the resulting curve with a Fermi function. We do not observe significant distortions of the spectra (other than shifts) that can be created by the highly nonuniform fields. From the magnitude of the shift, sample to analyzer distance, and electron kinetic energy, we place an upper limit on the magnetic field of $1 \mathrm{G}$ close to the sample surface. The EDCs are slightly shifted in energy as the sample is grounded via one of the current leads. The potential drop caused by the flow of current requires that the Fermi level-the zero of binding energy - be known at the point of measurement. This is achieved by measuring a reference spectrum at the $d$-wave node (Fermi crossing along the Brillouin zone diagonal), where the spectral gap is known to vanish at all temperatures, without moving the sample with respect to the photon beam. The leading edge of this gapless spectrum determines the zero of binding energy. If the potential drop were to occur at discrete weak links [12] in the superconductor, then we would expect to see multiple images of the spectrum displaced in voltage. We do not see any evidence for this in our data. On the other hand, if the potential drop occurs more or less uniformly across the sample, then we would see an inhomogeneous broadening of the ARPES spectrum, which is essentially equivalent to a degrading of the energy resolution. To minimize this broadening, we focus the photon beam to a fine spot $\sim 20 \mu \mathrm{m}$ in size along the current direction. (Detailed considerations of the effects of current, voltage, and magnetic field in ARPES experiment are discussed in Appendix A.)

One might also wonder if the large current density in the sample disturbs the electronic states. A simple Drude model estimate indicates that the change in momentum of the electrons due to the applied electric field is of order $10^{-4}$ of the Fermi momentum in the normal state, too small to be measured (as discussed in Appendix B). Another aspect of these experiments is Joule heating, once the sample enters the resistive regime [17,18]. Heating effects are discussed in detail in Appendixes C and D.

\section{B. Contrasting behavior of underdoped and overdoped cuprates}

A typical set of $I V$ curves for selected temperatures for an optimal doped $\left(T_{c}=90 \mathrm{~K}\right)$ sample is shown in Fig. 1(c). We use a constant voltage mode to prevent thermal runaway during the transition to the normal state. The curves are labeled by the temperature $T_{0}$ of the cold finger. Above $T_{c}$, at higher voltages the $I V$ curve deviates from a straight line (Ohmic) behavior due to heating. This deviation allows us to estimate the sample temperature when the voltage is applied (see Appendixes C and D). Below $T_{c}$, upon application of voltage, the current increases sharply and its slope is limited by the resistance of the invacuum wiring and contacts (we use a two-point contact method due to technical limitations). Coincidentally, this helps to limit the rapid onset of the current with voltage in the superconducting state and results in smooth $I V$ curves. When the current reaches a critical value, it peaks and then decreases with increasing voltage [19]. A crucial question is to what extent this negative differential resistance regime in the $I V$ characteristics arises simply due to Joule heating [18]. This has direct implications on whether the effects seen in the ARPES data in the presence of current flow are entirely due to heating effects, or if they are related to an interesting low-temperature resistive state generated by phase slips.

From the analysis presented in detail in Appendixes $\mathrm{C}$ and $\mathrm{D}$, we conclude that there indeed is an increase in the sample temperature above that of the cold finger; 
nevertheless, Joule heating alone cannot account for all of our observations. Specifically, we conclude that the increase in temperature in the presence of current flow still leaves the sample below $T_{c}$. This conclusion is based on analyzing the data using two separate methodologies and the simple fact that in this state the resistance of the samples is significantly below the value measured in the normal state for low currents. In Appendix C, we use a "pure heating model," which makes the worst-case assumption that the $I V$ is entirely dominated by heating. We show that this model is able to describe many aspects of the data, but not all. We argue that its shortcomings imply that this model overestimates the increase in temperature in the regime of interest. Next, in Appendix D, we directly estimate the rise in temperature using the measured $I V$ characteristics of the Bi2212 sample, together with the $I V$ of a thin layer of gold whose resistivity is similar to the normal state of Bi2212. We show that the estimated temperature remains well below $T_{c}$ in the regime of interest for our ARPES data.

In Fig. 2(a), we plot a schematic phase diagram of the cuprates and mark the locations where ARPES data are measured. In Fig. 2(b), we show the ARPES spectrum of an underdoped $\left(T_{c}=85 \mathrm{~K}\right)$ sample at the $(\pi, 0)$ point of the Brillouin zone as a function of the temperature. Upon increasing the temperature, the quasiparticle peak decreases in intensity and vanishes close to $T_{c}$ [7-9], while the pseudogap persists well above $T_{c}[3,10]$. At a low cold finger temperature, we drive the current through the sample by applying a voltage and measure the spectrum at the $(\pi, 0)$ point, as shown in Fig. 2(c). We use the spectrum at $(\pi, 0)$ rather than $k_{F}$ because it is easier to establish the presence of the coherent peak. The chemical potential is determined at each voltage by measuring the nodal spectrum, where the superconducting gap is zero. The currentvoltage values for each ARPES measurement are color coded on the $I V$ curve in Fig. 2(d). The top spectrum in Fig. 2(c) is measured without the current and is used as a reference. We start close to the peak of the $I V$ curve [Fig. 2(d)], which corresponds to the critical current for this cold finger temperature. The corresponding ARPES spectrum looks very similar to the reference, with a pronounced quasiparticle peak and superconducting gap. Since this spectrum is measured at the highest value of current, its similarity to the reference spectrum demonstrates that the magnetic field does not significantly affect the ARPES line shape. The presence of the coherent peak and superconducting gap in the spectrum also clearly demonstrates that the peak of the $I V$ curve corresponds to the vortex depinning [20] critical current rather than the depairing current. The ratio of the current flow velocity to the depairing velocity is very small; thus, the flow velocity (estimated to be at most $\sim 30 \mathrm{~m} / \mathrm{s}$ ) is 2 orders of magnitude smaller than the depairing velocity $\Delta / \hbar k_{F} \sim 9000 \mathrm{~m} / \mathrm{s}$, where $\Delta$ is the energy gap and $k_{F}$ the Fermi momentum
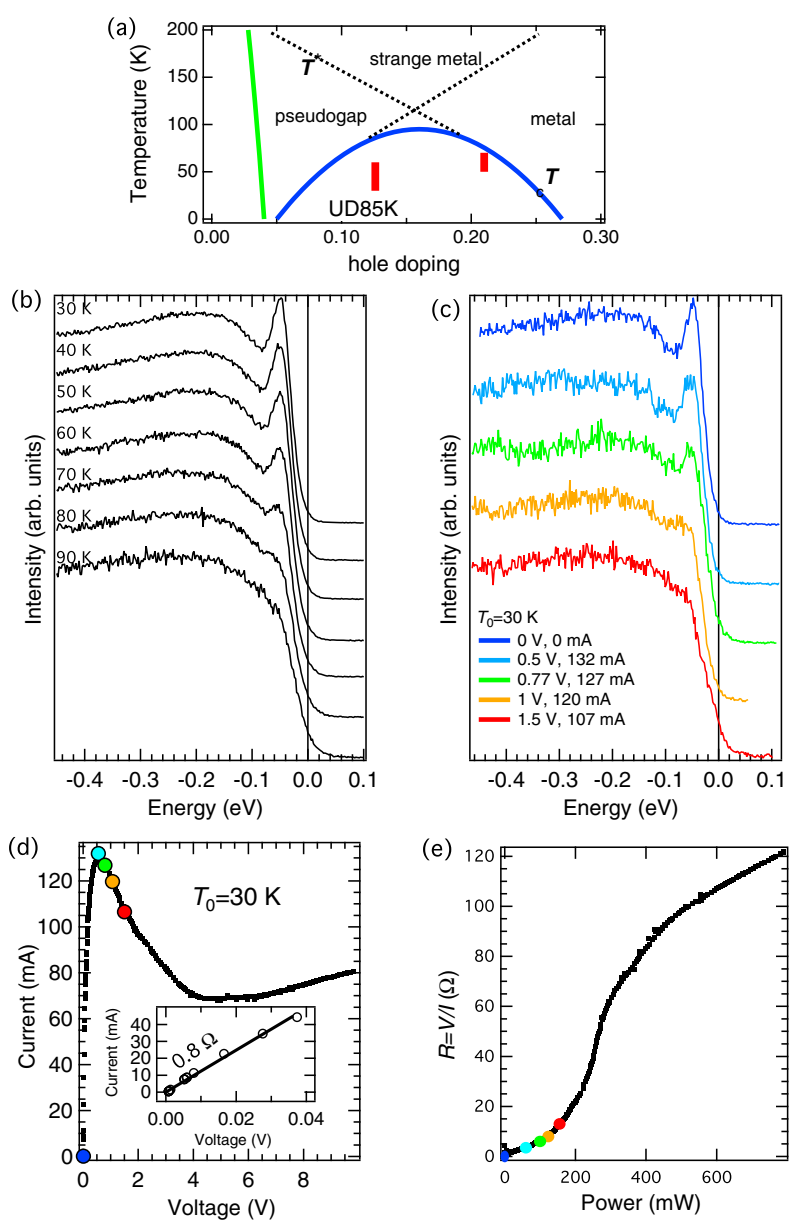

FIG. 2. Spectra at $(\pi, 0)$ and $I V$ characteristics for an underdoped $T_{c}=85 \mathrm{~K}$ sample. (a) Schematic phase diagram (after Ref. [6]) showing the doping and temperatures where current data are acquired (indicated by red bars). (b) $T$ dependence of the ARPES spectrum without current flow. (c) Low base temperature $(30 \mathrm{~K})$ spectrum with current passing through the sample for various voltages. (d) $I V$ curve for this sample. Colored circles indicate points where the ARPES data are acquired in (c). The inset shows a magnification of the low-voltage region. Since the sample is superconducting, the ratio of the voltage to current is equal to the resistance of the wiring and contacts, which for this sample is $0.8 \Omega$, much smaller than the normal state resistance. (e) $V / I$ versus the dissipated power. The colored circles mark points at which ARPES spectra are acquired in (c).

(see Appendix B). Surprisingly, as we increase the voltage, the quasiparticle peak decreases rapidly in intensity and it vanishes at a point where the current is significantly higher than the value observed above $T_{c}$. Even taking into account the heating (see Appendix D), we estimate the sample is still at $\sim 60 \mathrm{~K}$, well below $T_{c}$, at which temperature the coherent peak is still present in the absence of the current [Fig. 2(b)]. Thus, the loss of coherence observed in Fig. 2(c) corresponds to the onset of the resistance, rather than simply entering the normal state (i.e., due to heating alone), as is made clear in Fig. 2(e), which shows the variation of the resistance $(V / I)$ as a function of the 
(a)
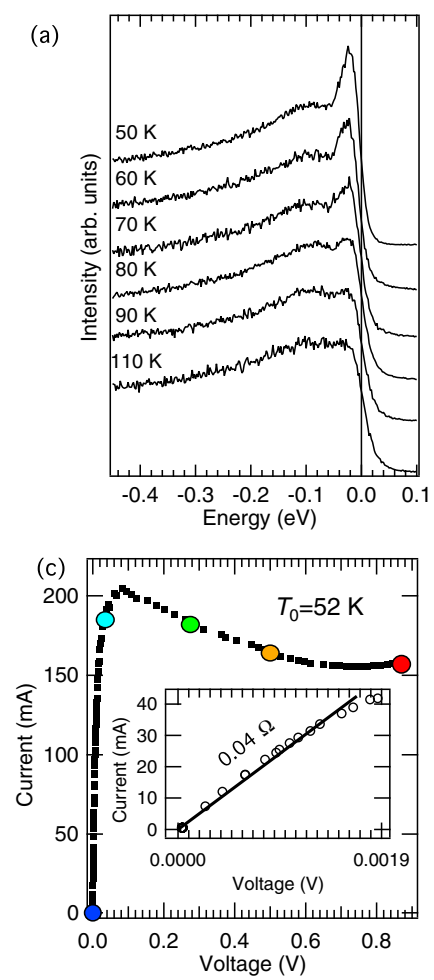

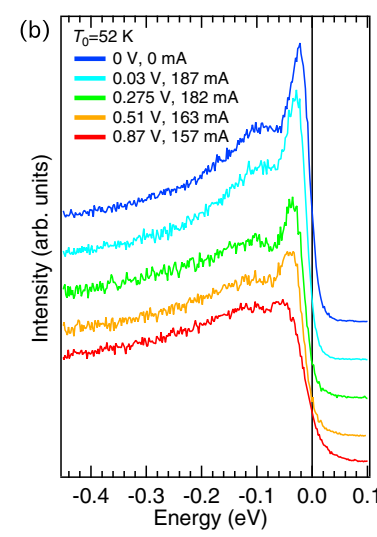

(d)

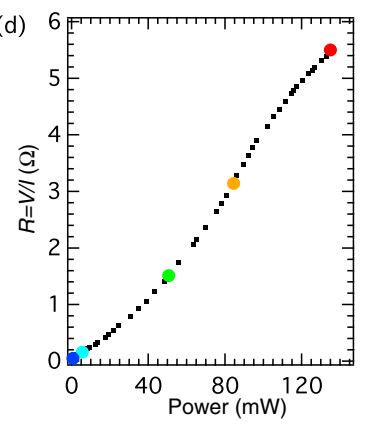

FIG. 3. Spectra at $(\pi, 0)$ and $I V$ characteristics for an overdoped $T_{c}=75 \mathrm{~K}$ sample. (a) $T$ dependence of the ARPES spectrum without current flow. (b) Low base temperature (52 K) spectrum with current passing through the sample for various voltages. (c) $I V$ curve for this sample. Colored circles indicate points where the ARPES data are acquired in (b). The inset shows a magnification of the low-voltage region. Since the sample is superconducting, the ratio of the voltage to current is equal to the resistance of the wiring and contacts, which for this sample is $0.04 \Omega$, much smaller than the normal state resistance. (d) $V / I$ versus the dissipated power. The colored circles mark points at which ARPES spectra are acquired in (b).

power $(I V)$. Clearly, the flow of current leads to extra dissipation, which in turn destroys single-particle coherence. Presumably, this extra dissipation is due to the development of phase slips and vortices $[17,21,22]$ rather than a loss of the pairing amplitude, as a well-defined energy gap is still present at the highest voltage.

We contrast this behavior with one of an overdoped $\left(T_{c}=75 \mathrm{~K}\right)$ sample shown in Fig. 3. In Fig. 3(a), we plot the temperature dependence of the spectrum at $(\pi, 0)$ measured with no current passing through the sample. The ARPES spectra shown in Fig. 3(b) are measured for several current-voltage values indicated on the $I V$ curve in Fig. 3(c). With increasing voltage, the quasiparticle peak decreases in intensity, but it remains visible even at the highest value of the voltage, where we have reached the normal state by a combination of the current flow and sample heating [the resistance of the sample at $0.87 \mathrm{~V}$, Fig. 3(d), being comparable to the dc resistance just above $T_{c}$ ].

\section{CONCLUSIONS}

In summary, by measuring ARPES in the presence of a transport current, we find that for underdoped samples, the loss of the quasiparticle peak occurs before reaching the normal state. Since the additional dissipation below $T_{c}$ due to current flow is thought to be due to phase slips and vortices, this indicates that superconducting phase fluctuations destroy the single-particle coherence, a very nonBCS-like behavior.

Our findings are of relevance to a microscopic understanding of high- $T_{c}$ superconductivity in the cuprates. Different ways of destroying superconductivity in the cuprates seem to lead to different "normal" states. For instance, raising the temperature above $T_{c}$ in zero magnetic field leads to a non-Fermi liquid state, with a pseudogap near the antinode with Fermi arcs near the nodes, whose origin is still being debated [23]. On the other hand, turning on a high magnetic field at low temperature leads to a Fermi liquid state with a Fermi surface reconstructed by broken translation symmetry [24].

Here, we propose a third route to destroying superconductivity by passing a current through the sample, and we address the question of single-particle coherence. In the future, it will be interesting to fully elucidate the nature of the resistive state arising from current flow. The experimental approach we present could be further developed by pulsing the current instead of working in a constant voltage mode, thus minimizing the heating $[25,26]$. We hope to report on such challenging experiments in the future.

\section{ACKNOWLEDGMENTS}

Work at Ames and Argonne was supported by the Materials Sciences and Engineering Division, Basic Energy Sciences, Office of Science, U.S. DOE. M. R. was supported by NSF Grant No. DMR-1410364. This work was based in part on data acquired at the Synchrotron Radiation Center, University of Wisconsin-Madison, which was supported by the National Science Foundation under Grant No. DMR-0537588.

\section{APPENDIX A: CHANGES OF THE KINETIC ENERGY AND MOMENTUM OF THE PHOTOELECTRONS DUE TO THE CURRENT AND VOLTAGE}

The presence of electric and magnetic fields between the sample and the electron analyzer affects the trajectories of the photoelectrons. In Fig. 1, we demonstrate that the magnetic field changes the angle of the photoelectrons without significantly affecting the spectral line shape. This can also be seen in Figs. 2(c) and 3(b), where the line shape at the peak current value is very similar to the one measured with no current.

To minimize the effects of the magnetic field, the return current is routed under the sample. For a single current 
element, the magnetic field decreases with distance as $1 / r^{2}$. Since we use two such elements with opposing currents, the resulting magnetic field decreases even faster $\left(\sim 1 / r^{3}\right)$. For example, if a 6-G field exists $1 \mathrm{~mm}$ from the sample, then $5 \mathrm{~mm}$ away the field is $\sim 100$ times smaller (i.e., $\sim 0.04 \mathrm{G}$ ) and a cyclotron radius $\sim 100$ times larger (i.e., $2 \mathrm{~m}$ ); $10 \mathrm{~mm}$ away this field is $\sim 1000$ times smaller and a cyclotron radius 1000 times larger (i.e., $20 \mathrm{~m}$ ). We therefore do not expect significant effects of the magnetic field on the spectra for modest values of the current.

The presence of a voltage drop across the sample has a more significant effect on the spectra. The voltage at a given point of the sample shifts the local chemical potential, which in turn affects the kinetic energy of the emitted photoelectrons. We demonstrate this in Fig. 4(a), where we plot EDCs at $k_{F}$ versus the actual kinetic energy. As the voltage is applied across the sample, the kinetic energy of the photoelectrons increases and the EDCs are shifted to the right. The amount of the shift is equal to the voltage that exists at the position on the sample from which
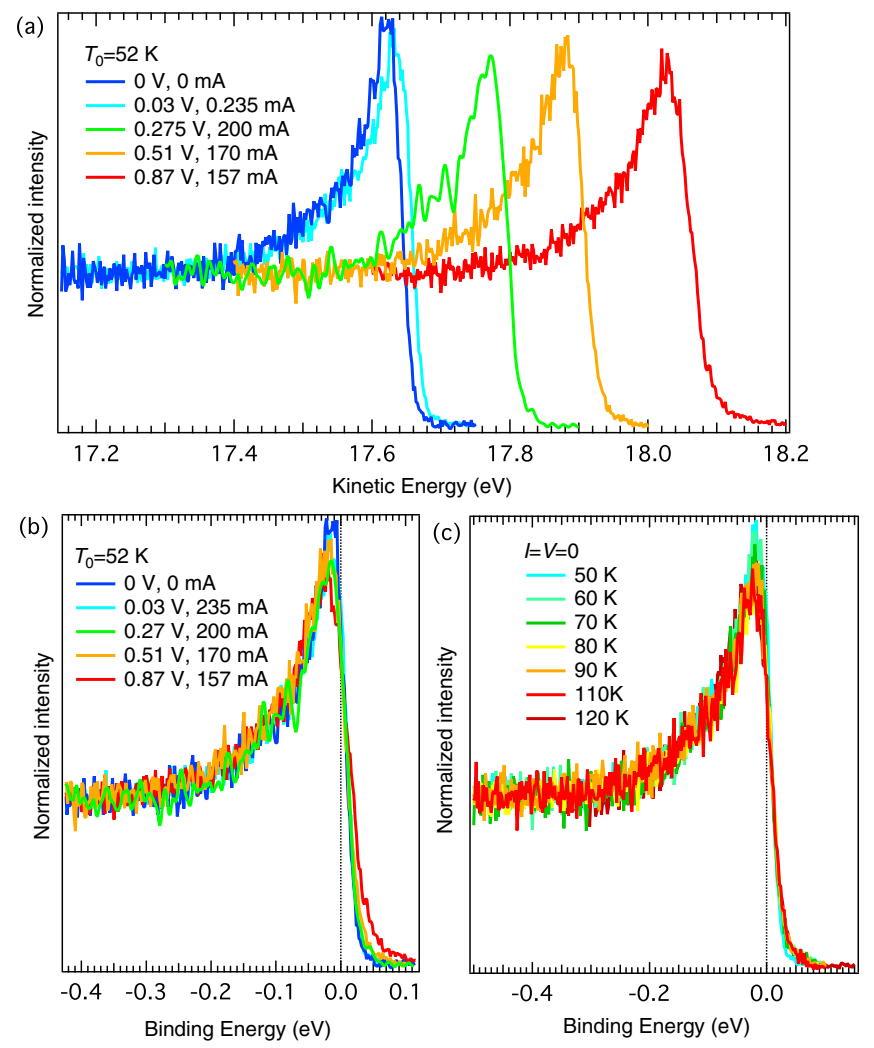

FIG. 4. EDCs at $k_{F}$ along the nodal direction for several voltages applied across the same sample as in Fig. 3, measured at a cold finger temperature $T_{0}=52 \mathrm{~K}$. (a) EDCs plotted as a function of the kinetic energy without any offsets. (b) EDCs plotted versus the binding energy. $E_{F}$ for each measurement is obtained by integrating the EDCs along the momentum cut and fitting the result with a Fermi function. (c) Zero current EDCs as a function of temperature. Note the contrast in the leading edge relative to that shown in (b). the photoelectrons are extracted. The value of the chemical potential for each voltage value can be obtained from such data measured at the node (where the superconducting gap is zero) by integrating the EDCs along the momentum cut and fitting the result with a Fermi function. The EDCs plotted versus binding energy after correcting for the changes of $E_{F}$ are shown in Fig. 4(b). The second effect of the voltage applied to the sample is a slight broadening of the spectra. This is because the photon beam has a finite size along the direction of the current flow and photoelectrons emitted within the spot size will have slightly different kinetic energies. This effect can be seen in Fig. 4(b), where at higher voltage values there is slight broadening of the leading edge, which is not evident in the zero current data, even at elevated temperatures [Fig. 4(c)]. To estimate this effect, let us assume that a potential drop of $1 \mathrm{~V}$ occurs uniformly across a 1-mm sample. Then the variation in the chemical potential across the $20 \mu \mathrm{m}$ beam width is $\delta \mu=20 \mathrm{meV}$, which is a significant effect. This leads to spectral broadening, i.e., $I(k, \omega)=$ $\int d \omega^{\prime} I_{0}\left(k, \omega-\omega^{\prime}\right)$, where the integration is over the window $-\delta \mu / 2<\omega^{\prime}<+\delta \mu / 2$ (here, $I$ is the measured intensity and $I_{0}$ the intensity without voltage broadening). In a sense, this is similar to the energy resolution convolution, and can be thought of as degrading the energy resolution. This broadening is roughly proportional to the ratio of the beam size along the direction of the current to the length of the bridge. This is perhaps the most challenging part of this experiment, as it requires the use of a very small photon beam to minimize the broadening effects, which in turn limits the photon flux and requires extended time for data acquisition.

\section{APPENDIX B: ESTIMATES OF CHANGES OF KEY QUANTITIES DUE TO FLOW OF CURRENT AND VALUE OF DEPAIRING CURRENT}

Shift in $k$ due to current flow.-The Drude model implies $\delta k=e \tau E / \hbar$. For $V=1$ volt across a 1-mm long sample, $e E=10^{3} \mathrm{eV} / \mathrm{m}$. For the relaxation time, one can estimate $\hbar / \tau=k_{B} T$, so that at $100 \mathrm{~K}$ one gets $\tau=10^{-13}$ s. Since $\tau$ increases below $T_{c}$, an upper bound estimate at $T=50 \mathrm{~K}$ is $\tau=10^{-12} \mathrm{~s}$. This yields $\delta k=1.5 \times 10^{6} \mathrm{~m}^{-1}$. Using $k_{F} \simeq \pi / a \simeq 10^{10} \mathrm{~m}^{-1}$ results in $\delta k / k_{F} \simeq 10^{-4}$, which is entirely negligible.

Flow velocity.-Assuming a uniform current distribution, a current of $125 \mathrm{~mA}$ through a cross-sectional area of $0.25 \mathrm{~mm} \times 500 \AA$ gives a current density $J=10^{10} \mathrm{~A} / \mathrm{m}^{2}$. Note that we assume the thinnest possible sample so that, if the actual sample thickness is more like 1000-1500 $\AA$, then the current density $J$ may well be smaller by a factor of $2-3$. The estimated flow velocity is $J=n e v$. The appropriate carrier density $n$ to use is not so obvious, but these uncertainties will not be of any importance, as we 
show below. If we use the smallest possible estimate $n \sim x$ (doped holes), then $n \simeq 0.25 /[3.83 \times 3.83 \times 7.5] \AA^{-3}$, where we use a value of $7.5 \AA$ as the average spacing between layers in Bi2212, so $n=2.25 \times 10^{27} \mathrm{~m}^{-3}$. This estimate of $n$ gives an estimated $v \simeq 30 \mathrm{~m} / \mathrm{s}$. Note that $v / v_{F} \sim 10^{-4}$ (where the experimental nodal $v_{F}$ is used). If, on the other hand, we assume 1 carrier per unit cell (as appropriate to $n \sim 1+x$ holes), then $n$ goes up by a factor of 5 , and, correspondingly, $v$ is reduced by a factor of 5 .

Depairing velocity.-As shown by Tinkham [27], the depairing velocity is reached when the Doppler-shifted spectrum becomes gapless. In our case, $v_{c}=\Delta / p_{F}$. Taking $\Delta=40 \mathrm{meV}$ and $p_{F}=\hbar k_{F} \quad\left(k_{F}\right.$ at the antinode is $0.67 \AA^{-1}$ ), we get $v_{c} \simeq 9000 \mathrm{~m} / \mathrm{s}$. So in the absolute "worst" case, our flow velocity is over 2 orders of magnitude less than the depairing velocity. A large difference between the drift velocity for the critical current and the depairing velocity is expected and also seen in classical superconductors [28]. This is reassuring because (a) the observed critical current is much smaller than the maximum possible theoretical value. and (b) if the pseudogap has anything to do with pairing, the current flows should not be large enough to cause depairing.

\section{APPENDIX C: ESTIMATION OF HEATING EFFECTS: PURE HEATING MODEL}

Applying a voltage across the sample will lead to Joule heating. Such heating will cause the sample temperature to vary as $T=T_{0}+I V / \kappa(T)$, where $T_{0}$ is the base temperature and $\kappa(T)$ the thermal conductance [18]. For simplicity, let us first assume that the thermal conductance is constant with $T$. Then, for a given voltage, the current is given by the condition $I=V / R\left(T_{0}+I V / \kappa\right)$, which can be determined by simple root finding. $R(T)$ is obtained from dc measurements of the resistance of the cleaved sample using a small constant current. In Fig. 5(a), we show simulated current versus voltage curves for various base temperatures using a $\kappa$ of $5 \mathrm{~mW} / \mathrm{K}$ in order to match the high-voltage data in Fig. 2(d). One sees a striking resemblance of these curves to those shown in Figs. 1(c) and 2(d), not only in shape and evolution as a function of base temperature, but also in the voltage location of the current maximum. The curves are not an exact match with Fig. 2(d), though. In particular, the current maximum is significantly larger in the simulated curves. In a pure heating model, this would be attributed to the $T$ dependence of $\kappa$.

To see this, one can fix $I$ from Fig. 2(d) and extract $\kappa(T)$. This is shown in Fig. 5(b), and has some resemblance to the $T$ dependence of the known thermal conductance of Bi2212 [29], but the inferred $\kappa$ drops by a factor of 10 when going from the normal to the superconducting state, unlike bulk Bi2212, which drops by only about a factor of 2 . We should remark that the actual thermal conductance should be limited by that of the STO substrate, which has
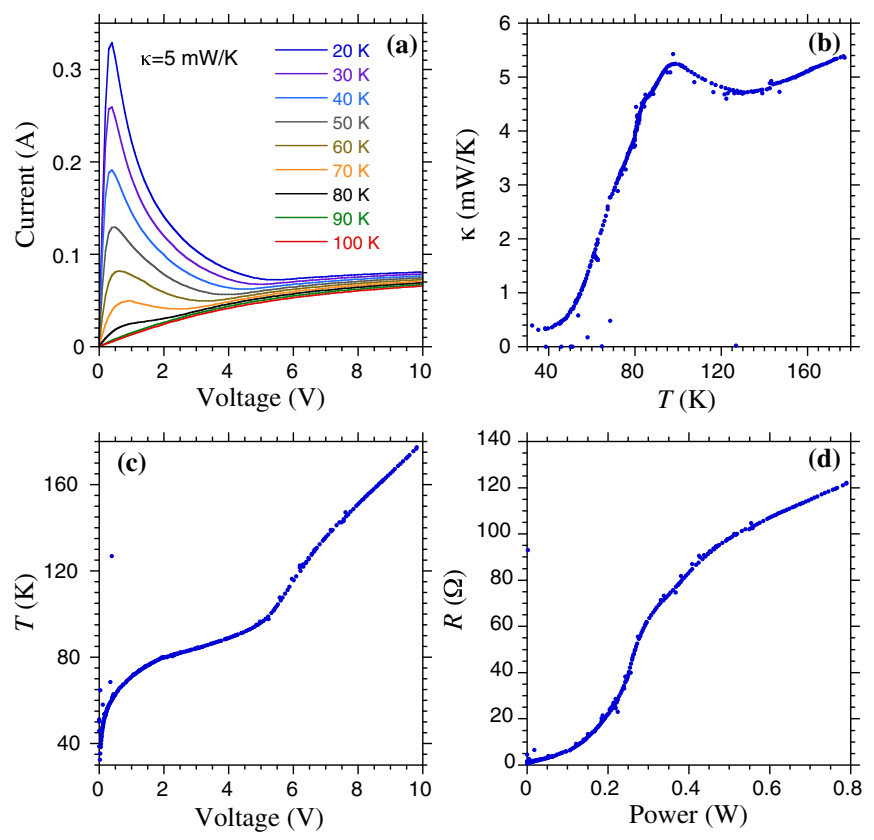

FIG. 5. A pure heating model analysis of the data in Fig. 2. (a) Simulated $I V$ curves for various base temperatures (assuming a thermal conductance $\kappa$ of $5 \mathrm{~mW} / \mathrm{K}$ ) obtained using the dc resistivity curve of the sample in Fig. 2. (b) $\kappa(T)$ obtained by fixing the current $I$ to that shown in Fig. 2(d). (c) Resulting variation of $T$ with the bias voltage. (d) Resistivity $(V / I)$ versus power $(I V)$, as in Fig. 2(e).

an even milder temperature dependence than Bi2212 [30]. This is verified by doing the same heating analysis for a $\mathrm{Au}$ film on STO (see Appendix D) at the same base temperature, which results in a weak temperature dependence for $\kappa$, giving rise to a nearly linear variation of $T$ with power.

From Fig. 5(b), one can plot $T$ versus the voltage, as shown in Fig. 5(c). In essence, one is using the sample as a thermometer. But the rapid rise of $T$ at low voltages seems unphysical, and is related to the unphysically large drop in $\kappa$ in Fig. 5(b). This is also evident in Fig. 5(d), where we plot $R$ versus the power, $I V$. The development of resistance with power is more dramatic than the development of the dc resistance with temperature [Fig. 7(b)]. This implies, as discussed in the main text, that there is extra dissipation below $T_{c}$ due to the current flow.

\section{APPENDIX D: EMPIRICAL ESTIMATE OF HEATING}

The actual sample temperature above $T_{c}$ under current flow is relatively easy to determine, as discussed in Appendix C. To accomplish this, we use the sample itself as a thermometer, utilizing the temperature dependence of the dc resistance. To illustrate this, we plot the $I V$ curves measured for cold finger temperatures above $T_{c}$ in Fig. 6. The dotted curves are the actual measured $I V$. The straight lines are fits to the low-voltage data and represent ideal $I V$ 's 


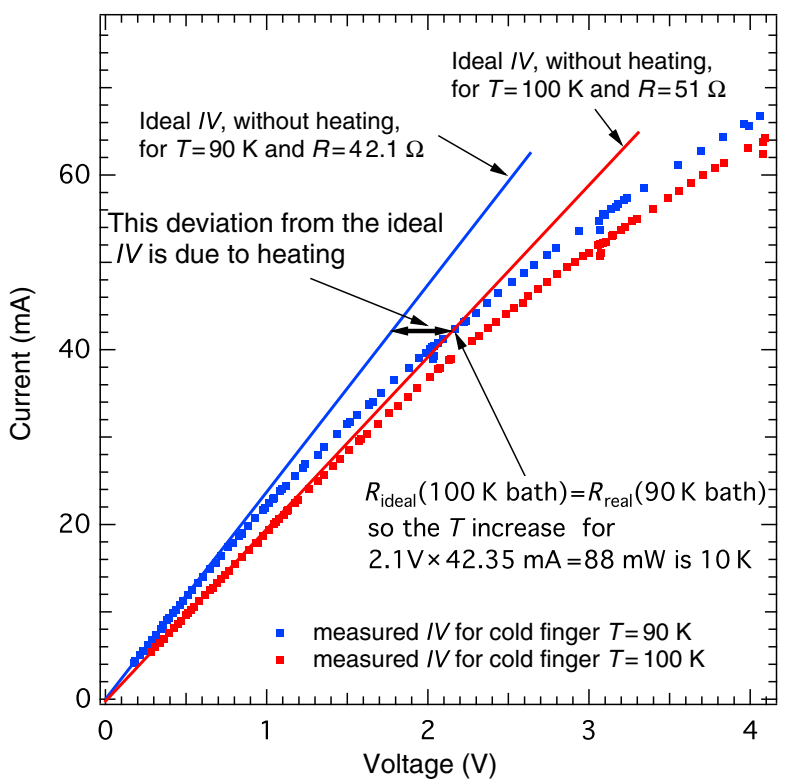

FIG. 6. $\quad I V$ curves above $T_{c}$ from Fig. 1(c). Straight lines are fits to the low-voltage part and signify ideal $I V$ curves (with a slope equal to the inverse of the resistance) of the sample in the absence of heating.

in the absence of heating, with their slopes being equal to the inverse resistance. The actual $I V$ for $T_{0}=90 \mathrm{~K}$ (blue dots) crosses the ideal $T_{0}=100 \mathrm{~K}$ (red line) at $\sim 2.1 \mathrm{~V}$ $(P=88 \mathrm{~mW})$; therefore, at this power dissipation the actual sample temperature is $T=100 \mathrm{~K}$ (a heating of $11.4 \mathrm{~K} / 100 \mathrm{~mW}$ ). A more systematic approach is to use the $R$ versus power plot obtained from the $I V$ curves, and then convert the resistance for a given power to temperature using the temperature dependence of the resistance. This is demonstrated in Fig. 7. At $200 \mathrm{~mW}$ and a cold finger temperature of $T_{0}=120 \mathrm{~K}$, the resistance of the sample (obtained by dividing the voltage by the current) is $66.6 \Omega$ [Fig. 7(a)]. This value of the resistance corresponds to a temperature of $149.2 \mathrm{~K}$ on the $R$ versus $T$ curve shown in Fig. 7(b). Therefore, a dissipation of $200 \mathrm{~mW}$ causes a heating of the sample by $29.2 \mathrm{~K}(14.6 \mathrm{~K} / 100 \mathrm{~mW})$, a bit higher than the result at $T_{0}=90 \mathrm{~K}$. The $R$ versus power curve can also be directly converted to an actual sample temperature versus power. This is done by again utilizing the $R$ versus $T$ curve. The result is shown in Fig. 7(c) for various base temperatures, and allows one to directly read off the actual temperature of the sample for any power dissipation within the measured range.

The estimation of the sample temperature for cold finger temperatures below $T_{c}$ is more complicated because of the presence of the superfluid. It is difficult to separate the changes of the resistance due to heating from those due to phase slips, as discussed in Appendix C. Furthermore, although the thermal conductance of Bi2212 does decrease below $T_{c}$ [29], since the thickness of the sample is only $\sim 500 \AA$ and therefore 5000 times smaller than that of the
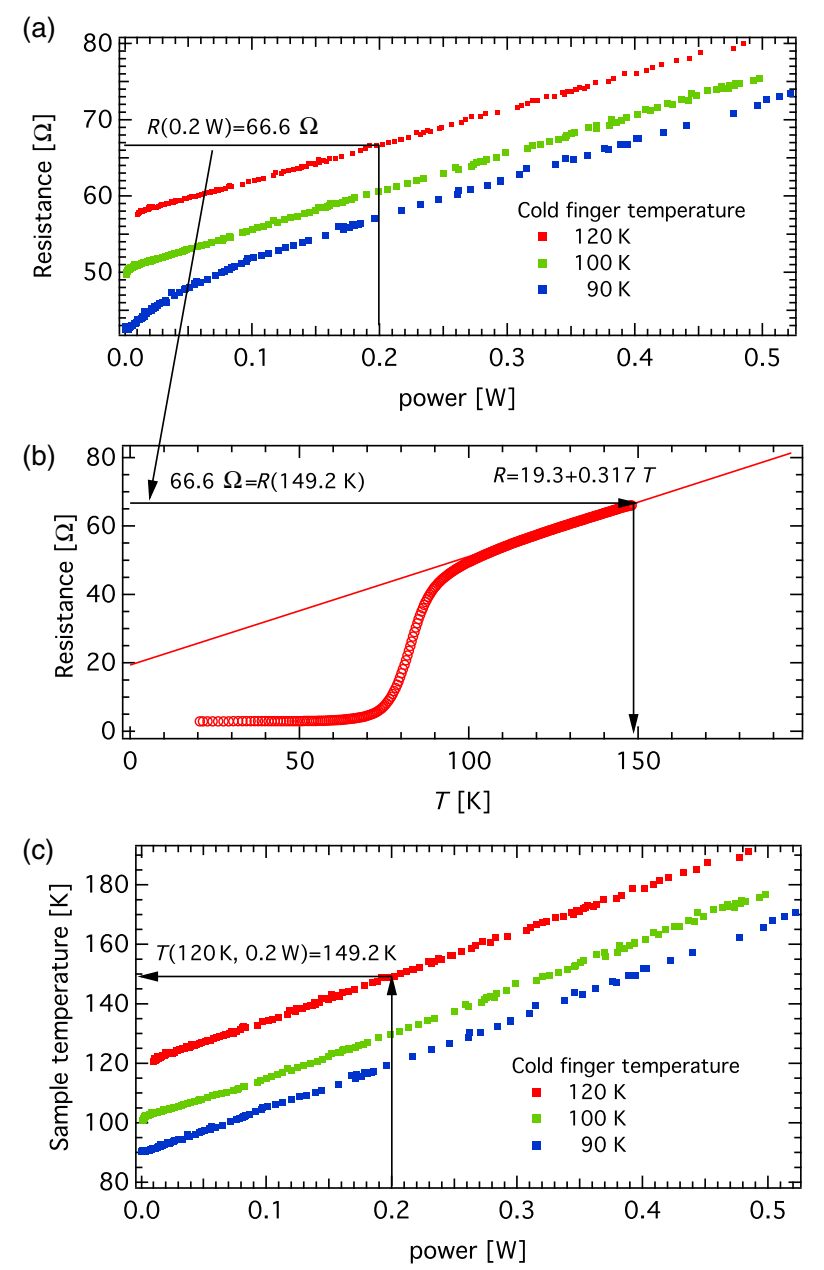

FIG. 7. Estimation of the sample temperature in the presence of voltage using data for OP Bi2212, the same as in Fig. 1(c). (a) Resistance versus power for three temperatures of the cold finger. (b) dc resistance versus temperature. (c) Actual sample temperature versus power calculated by converting the data in (a) using the dc resistance shown in (b).

STO substrate, we can safely assume that the vast majority of the thermal gradient occurs across the STO substrate, interfaces [31], and epoxy with which the substrate is attached to the copper block. The thermal conductivity of STO peaks at $\sim 17 \mathrm{~W} / \mathrm{m} \times \mathrm{K}$ at $100 \mathrm{~K}$ and decreases to $\sim 10 \mathrm{~W} / \mathrm{m} \times \mathrm{K}$ at $40 \mathrm{~K}$ [30]. The thermal conductance of the epoxy is difficult to locate, but its thickness is smaller than the one of the STO substrate by at least an order of magnitude. Even though the presence of various interfaces, the substrate, and the epoxy makes this a complicated heat transfer system, the thermal gradient across it can be easily determined experimentally at low temperatures by utilizing a nonsuperconducting film. To do this, we carefully strip the Bi2212 film, clean the substrate, and evaporate a thin layer of gold so that its resistivity is similar to the one in the normal state of Bi2212. We can then study the thermal resistance of the substrate at low cold finger temperatures in a similar fashion as described above. In Fig. 8(a), we plot 

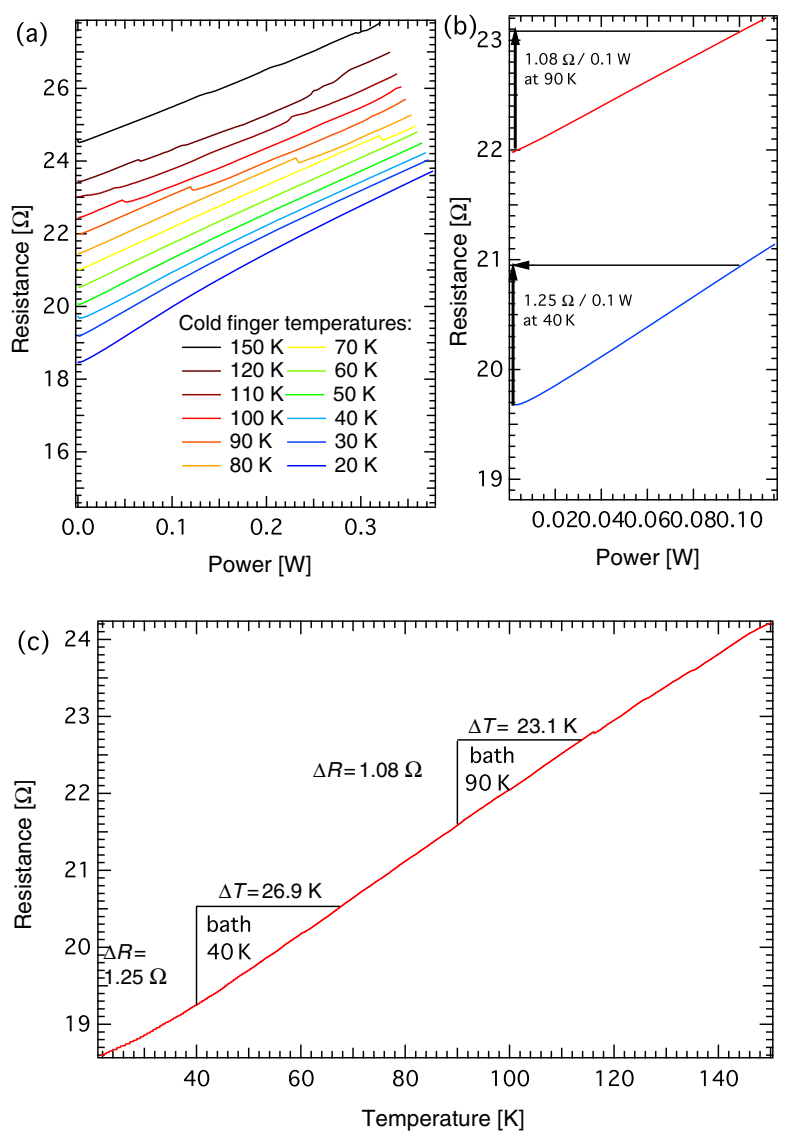

FIG. 8. Heating effects at low and high temperatures in a gold film evaporated on the STO substrate under conditions similar to ones in Fig. 2. (a) $R$ versus power for various cold finger temperatures. (b) $R$ versus power for two temperatures of the cold finger showing the values of the increase of the resistance with power dissipation. (c) $R$ versus $T$ curve used for converting the increase of the resistance to an increase of the temperature.

the resistance versus power curves for a number of cold finger temperatures. In Fig. 8(b), we focus on two values of $T_{0}=40$ and $90 \mathrm{~K}$. At $100-\mathrm{mW}$ dissipation, the increase of the resistance is 1.25 and $1.08 \Omega$, respectively, which can be converted to an increase of the sample temperature using the $R$ versus $T$ curve shown in Fig. 8(c). We find that at $T_{0}=40 \mathrm{~K}$, the sample heating is $27 \mathrm{~K}$ per $100 \mathrm{~mW}$, and for $T_{0}=90 \mathrm{~K}$, it is $23 \mathrm{~K}$ per $100 \mathrm{~mW}$. The decrease of the thermal conductance at low temperatures is consistent with data available for STO, where there is a peak in the thermal conductance at $\sim 100 \mathrm{~K}[30]$. As mentioned in Appendix C, use of a pure heating analysis leads to a mild increase of the thermal conductance by $18 \%$ at a base temperature of $30 \mathrm{~K}$ when the power increases from 0 to $370 \mathrm{~mW}$.

In Fig. 9, we compare the $I V$ curves, $V / I$ ratio versus voltage, and $V / I$ ratio versus power for the data of Fig. 1. For base temperatures above $T_{c}$, the $V / I$ versus voltage curves, shown in Fig. 9(b), are nonlinear due to heating (as demonstrated in Fig. 6). For base temperatures below $T_{c}$, there is a change of slope [marked by arrows in Fig. 9(b)]
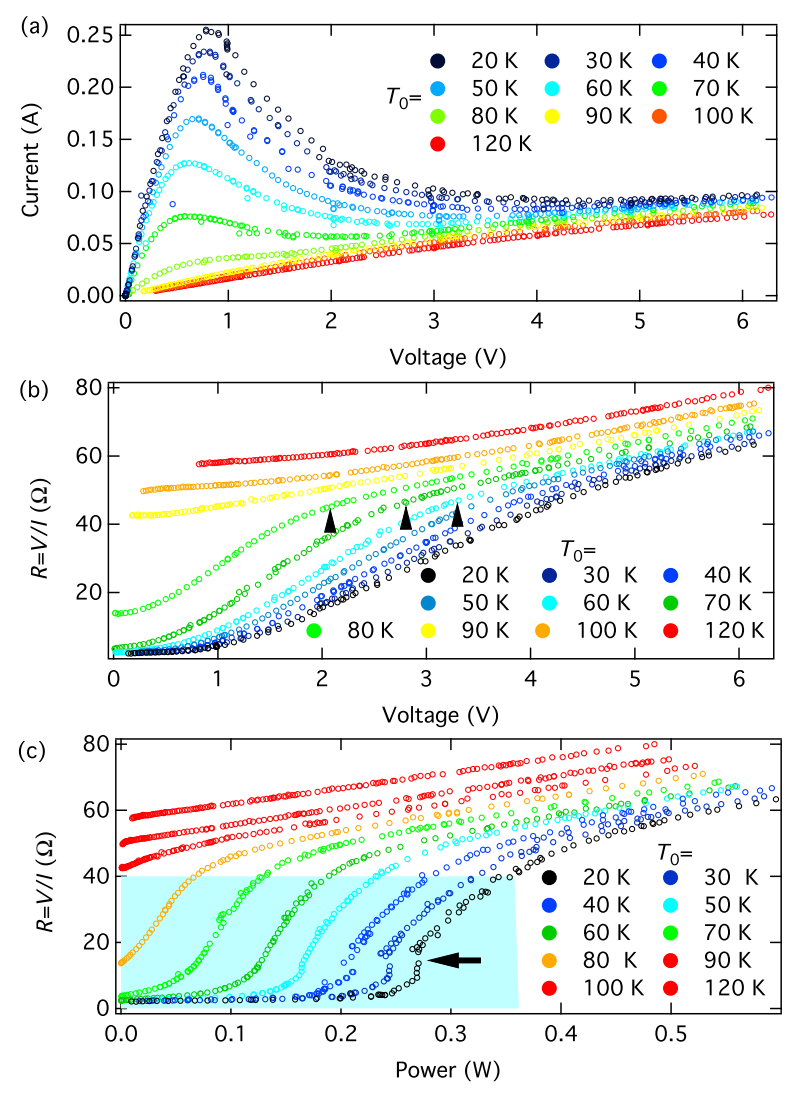

FIG. 9. Transport data for the UD85K sample. (a) IV curves [same as in Fig. 1(c)]. (b) $V / I$ ratio versus voltage. Arrows mark a change in slope for the temperatures indicated. (c) $V / I$ ratio versus power. The blue shaded rectangle marks the area where the $V / I$ ratio deviates from the normal state linear in power behavior, indicating the presence of superconductivity.

that indicates the presence of superconductivity for lower voltage values. As demonstrated in Fig. 7, the $V / I$ ratio for base temperatures above $T_{c}$ depends linearly on power. Such a plot therefore better reveals the presence of superconductivity at lower voltage values, and is shown in Fig. 9(c). For base temperatures of $80 \mathrm{~K}$ and below, there is a deviation from the normal state linear behavior with power even for power values as high as $350 \mathrm{~mW}$. This drop in the $V / I$ ratio as compared to a linear extrapolation from higher voltages is caused by the presence of superconductivity, and provides evidence that even at such an elevated power, the sample temperature is below $T_{c}$ within the area marked by the blue shaded rectangle.

\section{APPENDIX E: COMPARISON OF TWO-POINT CONTACT AND FOUR-POINT CONTACT MEASUREMENTS OF THE IV CURVES}

Because of technical limitations, we utilize two-point contact measurements of the $I V$ curves for the samples used in our ARPES experiments. It is important to verify the relation of such $I V$ curves to ones measured in a 
"proper" four-point contact geometry, where the voltage is measured across the sample itself, excluding any drop at the sample-contact interface. To accomplish this, we prepare a sample with contacts in standard four-point geometry and simultaneously measure the current, voltage drop across the contacts $\left(V_{c}\right)$, and sample $\left(V_{s}\right)$. The measurements are conducted in "constant" voltage mode, where we control the voltage across the contacts and the current is limited by the resistance of the sample and contacts. This prevents thermal runaway that commonly occurs in constant current measurements due to a rapid increase of the voltage and dissipated power as the sample transitions into the resistive state [13]. Based on such data, we can measure the $I V$ curves for two-point contact and four-point contact geometries at the same time under the same sample temperature, dissipated power, and current conditions. We apply a known voltage $V_{c}$ to the outer contacts, and for each of its values we measure the current $I$ and voltage on the inner electrodes $V_{s}$. The results are shown in Fig. 10. The twopoint contact $I V$ curve ( $I$ versus $V_{c}$ ) is plotted in red and is similar to data in the previous figures. The four-point contact $I V$ curve $\left(I\right.$ versus $\left.V_{s}\right)$ is plotted in blue. With double-ended arrows, we show examples of $V_{c}$ and $V_{s}$ pairs that are measured at the same time for the same current value. We first note that in the "negative resistance" regime (right side of the current maximum) both curves are qualitatively similar. The current decreases with increasing

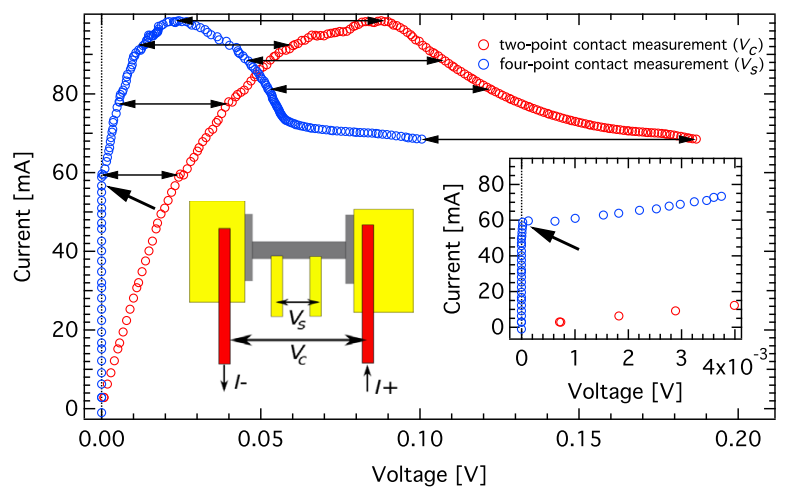

FIG. 10. Comparison of $I V$ curves measured using a two-point and a four-point contact method at a cold finger temperature of $70 \mathrm{~K}$ and an optimally doped sample with $T_{c}=90 \mathrm{~K}$. The voltage for the red curve $\left(V_{c}\right)$ is measured across the outer current contacts, and the voltage for the blue curve $\left(V_{s}\right)$ is measured across two separate electrodes connected to the mid section of the bridge that do not have direct electrical contact with the current electrodes other than through the sample. The single arrow indicates the value of the current at the onset of the resistive transition. The black double arrows connect pairs of $V_{c}$ and $V_{s}$ points that are measured at the same time. The left-hand inset shows a drawing of the measurement geometry. The sample is shown in gray, gold contacts in yellow, and copper leads in red. The silver paste linking the copper leads and gold contacts is not shown for clarity. The right-hand inset shows the expanded lowvoltage region. voltage and reaches a minimum for higher voltage values. For our purposes, this is the most important point, since all the ARPES data we show in this paper are taken in this regime. At very low voltages, there is, of course, a more significant difference between the two curves. In a fourpoint contact measurement, the voltage is measured across a small section of the bridge and does not include the potential drop across the contacts. In the two-point contact case, the voltage is measured across the whole bridge and contacts. Therefore, $V_{s}$ will remain zero up to a certain value of the current (signifying that the sample is in the zero resistance state). The value of $V_{s}$ will be lower than $V_{c}$ for the same current, as this drop is measured over a shorter portion of the bridge and does not include the contact resistance. The current will initially increase linearly with $V_{c}$ voltage, which reflects the resistance of the contacts and wires. This behavior is consistent with the insets of Figs. 2(d) and 3(c), validating that part of the discussion of the $I V$ curves.

[1] J. Bardeen, L. N. Cooper, and J. R. Schrieffer, Theory of Superconductivity, Phys. Rev. 108, 1175 (1957).

[2] H. Ding, T. Yokoya, J. C. Campuzano, T. Takahashi, M. Randeria, M. R. Norman, T. Mochiku, K. Kadowaki, and J. Giapintzakis, Spectroscopic Evidence for a Pseudogap in the Normal State of Underdoped High- $T_{c}$ Superconductors, Nature (London) 382, 51 (1996).

[3] A. G. Loeser, Z.-X. Shen, D. S. Dessau, D. S. Marshall, C. H. Park, P. Fournier, and A. Kapitulnik, Excitation Gap in the Normal State of Underdoped $\mathrm{Bi}_{2} \mathrm{Sr}_{2} \mathrm{CaCu}_{2} \mathrm{O}_{8+\delta}$, Science 273, 325 (1996).

[4] M. R. Norman, H. Ding, M. Randeria, J. C. Campuzano, T. Yokoya, T. Takeuchi, T. Takahashi, T. Mochiku, K. Kadowaki, P. Guptasarma, and D. G. Hinks, Destruction of the Fermi Surface Underdoped High- $T_{c}$ Superconductors, Nature (London) 392, 157 (1998).

[5] A. Kaminski, S. Rosenkranz, H. M Fretwell, Z.Z. Li, H. Raffy, M. Randeria, M. R. Norman, and J. C. Campuzano, Crossover from Coherent to Incoherent Electronic Excitations in the Normal State of $\mathrm{Bi}_{2} \mathrm{Sr}_{2} \mathrm{CaCu}_{2} \mathrm{O}_{8+\delta}$, Phys. Rev. Lett. 90, 207003 (2003).

[6] U. Chatterjee, D. Ai, J. Zhao, S. Rosenkranz, A. Kaminski, H. Raffy, Z.Z. Li, K. Kadowaki, M. Randeria, M. R. Norman, and J. C. Campuzano, Electronic Phase Diagram of High-Temperature Copper Oxide Superconductors, Proc. Natl. Acad. Sci. U.S.A. 108, 9346 (2011).

[7] M. Randeria, H. Ding, J. C. Campuzano, A. Bellman, G. Jennings, T. Yokoya, T. Takahashi, H. Katayama-Yoshida, T. Mochiku, and K. Kadowaki, Momentum Distribution Sum Rule for Angle-Resolved Photoemission, Phys. Rev. Lett. 74, 4951 (1995).

[8] A. V. Fedorov, T. Valla, P. D. Johnson, Q. Li, G. D. Gu, and N. Koshizuka, Temperature Dependent Photoemission Studies of Optimally Doped $\mathrm{Bi}_{2} \mathrm{Sr}_{2} \mathrm{CaCu}_{2} \mathrm{O}_{8+\delta}$, Phys. Rev. Lett. 82, 2179 (1999).

[9] D. L. Feng, D. H. Lu, K. M. Shen, C. Kim, H. Eisaki, A. Damascelli, R. Yoshizaki, J.-i. Shimoyama, K. Kishio, 
G. D. Gu, S. Oh, A. Andrus, J. O’Donnell, J. N. Eckstein, and Z.-X. Shen, Signature of Superfluid Density in the Single-Particle Excitation Spectrum of $\mathrm{Bi}_{2} \mathrm{Sr}_{2} \mathrm{CaCu}_{2} \mathrm{O}_{8+\delta}$, Science 289, 277 (2000).

[10] H. Ding, J. R. Engelbrecht, Z. Wang, J. C. Campuzano, S.-C. Wang, H.-B. Yang, R. Rogan, T. Takahashi, K. Kadowaki, and D. G. Hinks, Coherent Quasiparticle Weight and Its Connection to High- $T_{c}$ Superconductivity from AngleResolved Photoemission, Phys. Rev. Lett. 87, 227001 (2001).

[11] L. Goren and E. Altman, Quenching the Superconducting State of Cuprate Compounds with Electric Currents: A Variational Study, Phys. Rev. Lett. 104, 257002 (2010).

[12] K. E. Gray, A New Inhomogeneous State of Superconducting Films, J. Low Temp. Phys. 23, 679 (1976).

[13] M. Naamneh, J. C. Campuzano, and A. Kanigel, Doping Dependence of the Critical Current in $\mathrm{Bi}_{2} \mathrm{Sr}_{2} \mathrm{CaCu}_{2} \mathrm{O}_{8+\delta}$, Phys. Rev. B 90, 224501 (2014).

[14] V. A. Komashko, A. G. Popov, V. L. Svetchnikov, A. V. Pronin, V. S. Melnikov, A. Y. Galkin, V. M. Pan, C. L. Snead, and M. Suenaga, Critical Current Density of Thin YBCO Films on Buffered Sapphire Substrates, Supercond. Sci. Technol. 13, 209 (2000).

[15] L. J. MacManus-Driscoll, S. R. Foltyn, Q. X. Jia, H. Wang, A. Serquis, L. Civale, B. Maiorov, M. E. Hawley, M. P. Maley, and D. E. Peterson, Strongly Enhanced Current Densities in Superconducting Coated Conductors of $\mathrm{YBa}_{2} \mathrm{Cu}_{3} \mathrm{O}_{7-x}+\mathrm{BaZrO}_{3}$, Nat. Mater. 3, 439 (2004).

[16] E. F. Talantsev and J. L. Tallon, Universal Self-Field Critical Current for Thin-Film Superconductors, Nat. Commun. 6, 7820 (2015).

[17] W. J. Skocpol, M. R. Beasley, and M. Tinkham, SelfHeating Hotspots in Superconducting Thin-Film Microbridges, J. Appl. Phys. 45, 4054 (1974).

[18] V. N. Zavaritsky, Joule Heating versus 'Intrinsic' Tunnelling in HTSC, Physica (Amsterdam) 404C, 440 (2004).

[19] D. Y. Vodolazov, F. M. Peeters, L. Piraux, S. MatefiTempfli, and S. Michotte, Current-Voltage Characteristics of Quasi-One-Dimensional Superconductors: An S-Shaped Curve in the Constant Voltage Regime, Phys. Rev. Lett. 91, 157001 (2003).

[20] O. M. Auslaender, L. Luan, E. W. J. Straver, J. E. Hoffman, N. C. Koshnick, E. Zeldov, D. A. Bonn, R. Liang, W. N. Hardy, and K. A. Moler, Mechanics of Individual Isolated Vortices in a Cuprate Superconductor, Nat. Phys. 5, 35 (2009).
[21] M. R. Beasley, J. E. Mooij, and T. P. Orlando, Possibility of Vortex-Antivortex Pair Dissociation in Two-Dimensional Superconductors, Phys. Rev. Lett. 42, 1165 (1979).

[22] S. Martin, A. T. Fiory, R. M. Fleming, G. P. Espinosa, and A. S. Cooper, Vortex-Pair Excitation near the Superconducting Transition of $\mathrm{Bi}_{2} \mathrm{Sr}_{2} \mathrm{CaCu}_{2} \mathrm{O}_{8}$ Crystals, Phys. Rev. Lett. 62, 677 (1989).

[23] B. Keimer, S. A. Kivelson, M. R. Norman, S. Uchida, and J. Zaanen, From Quantum Matter to High-Temperature Superconductivity in Copper Oxides, Nature (London) 518, 179 (2015).

[24] N. Doiron-Leyraud, C. Proust, D. LeBoeuf, J. Levallois, J.-B. Bonnemaison, R. Liang, D. A. Bonn, W. N. Hardy, and L. Taillefer, Quantum Oscillations and the Fermi Surface in an Underdoped High- $T_{c}$ Superconductor, Nature (London) 447, 565 (2007).

[25] K. Anagawa, Y. Yamada, T. Shibauchi, M. Suzuki, and T. Watanabe, 60 ns Time Scale Short Pulse Interlayer Tunneling Spectroscopy of $\mathrm{Bi}_{2} \mathrm{Sr}_{2} \mathrm{CaCu}_{2} \mathrm{O}_{8+\delta}$, Appl. Phys. Lett. 83, 2381 (2003).

[26] W. Lang, I. Puica, K. Siraj, M. Peruzzi, J. D. Pedarnig, and D. Buerle, Critical Current Enhancement in $\mathrm{YBa}_{2} \mathrm{Cu}_{3} \mathrm{O}_{7-\delta}$ towards the Intrinsic Depairing Value in Short Current Pulses, Physica (Amsterdam) 460-462C, 827 (2007).

[27] M. Tinkham, Introduction to Superconductivity (McGrawHill, New York, 1996).

[28] C. Buzea and T. Tamashita, Review of the Superconducting Properties of $\mathrm{MgB}_{2}$, Supercond. Sci. Technol. 14, R115 (2001).

[29] Y. Ando, J. Takeya, Y. Abe, K. Nakamura, and A. Kapitulnik, Temperature- and Magnetic-Field-Dependent Thermal Conductivity of Pure and $\mathrm{Zn}$-Doped $\mathrm{Bi}_{2} \mathrm{Sr}_{2} \mathrm{CaCu}_{2} \mathrm{O}_{8+\delta}$ Single Crystals, Phys. Rev. B 62, 626 (2000).

[30] C. Yu, M. L. Scullin, M. Huijben, R. Ramesh, and A. Majumdar, Thermal Conductivity Reduction in OxygenDeficient Strontium Titanates, Appl. Phys. Lett. 92, 191911 (2008).

[31] J. Ravichandran, A. K. Yadav, R. Cheaito, P. B. Rossen, A. Soukiassian, S. J. Suresha, J. C. Duda, B. M. Foley, C.-H. Lee, Y. Zhu, A. W. Lichtenberger, J. E. Moore, D. A. Muller, D. G. Schlom, P. E. Hopkins, A. Majumdar, R. Ramesh, and M. A. Zurbuchen, Crossover from Incoherent to Coherent Phonon Scattering in Epitaxial Oxide Superlattices, Nat. Mater. 13, 168 (2014). 